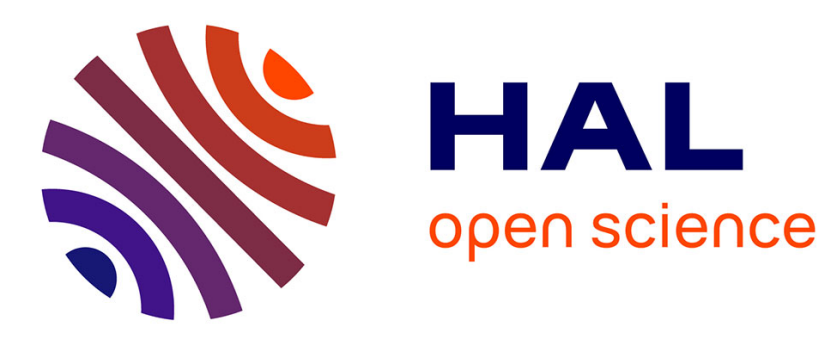

\title{
Solubility of gas in confined systems. Nonextensive thermodynamics approach.
}

Pierre Letellier, Mireille Turmine

\section{To cite this version:}

Pierre Letellier, Mireille Turmine. Solubility of gas in confined systems. Nonextensive thermodynamics approach.. Journal of Colloid and Interface Science, 2013, 392, pp.382-387. 10.1016/j.jcis.2012.10.009 . hal-00803267

\section{HAL Id: hal-00803267 \\ https://hal.sorbonne-universite.fr/hal-00803267}

Submitted on 4 Mar 2015

HAL is a multi-disciplinary open access archive for the deposit and dissemination of scientific research documents, whether they are published or not. The documents may come from teaching and research institutions in France or abroad, or from public or private research centers.
L'archive ouverte pluridisciplinaire HAL, est destinée au dépôt et à la diffusion de documents scientifiques de niveau recherche, publiés ou non, émanant des établissements d'enseignement et de recherche français ou étrangers, des laboratoires publics ou privés. 


\title{
Solubility of gas in confined systems.
}

\section{Non Extensive Thermodynamics approach}

\author{
Pierre Letellier, Mireille Turmine* \\ Laboratoire Interfaces et Systèmes Electrochimiques, \\ CNRS, UPR15 LISE, \\ Université Pierre et Marie Curie Paris 6, Case 133, \\ 4 place Jussieu, 75252 Paris Cedex 05, France \\ e-mail: mireille.turmine@upmc.fr
}

\begin{abstract}
The use of the concepts of the nonextensive thermodynamics allows reconsidering the equilibrium of bubble solubilization, and more commonly of gaseous aggregates in supersaturated solutions of gas. The introduced relations are general and include as particular cases the equations usually used to describe these phenomena. These equations are discussed. Especially, we specified the domain of application of Kelvin's relation which was illustrated by the solubility of gases in fogs and clouds. Various possibilities of thoughts on the behavior of the gaseous aggregates and nano-systems are proposed. Thus, the introduced relations permit to consider the presence of gaseous aggregates in equilibrium with the solution even for undersaturated solution. Non-extensive thermodynamics admits the notion of negative pressure at the inner of confined phases (solid or liquid).
\end{abstract}

Keywords: bubbles, nano-bubbles, sessile bubbles, thermodynamics, nonextensive thermodynamics, solubility, negative pressure, Henry's law, Laplace's law. 


\section{Introduction}

The behavior of fluids supersaturated in gases concerns numerous scientific domains such as geologic field with the formation of bubbles in magmas $[1,2]$, in industrial domain with the obtaining of structured materials (foams, micro-cellular materials), in food-processing industries (soda drinks [3]), in water treatment [4], surface cleaning [5] or medicine [6]. These situations are particularly studied for the establishment of the procedures of decompression of the persons working in hyperbaric atmosphere (divers, deep-sea divers) [712], or for the understanding of the breathe of marine species [13]. Their study is therefore of the highest importance by their benefits. There are many theoretical approaches of this subject [14], but as S. Lubetkin [15] pointed out, rarely the experimental results can confirm their relevance. His judgment on this point is interesting: "This disagreement is on two fronts: (1) first, quantitative, in that theory predicts much higher supersaturations are needed to cause bubble nucleation than are found necessary experimentally (characteristically, a factor of at least 2 orders of magnitude in the supersaturation); (2) second, qualitative, in that theory predicts that it is only the nature of the liquid which dictates the conditions required for gas bubble nucleation. Experimentally, the chemical nature of the gas is found to be important". Our goal in this paper is to sum up on this subject, but also to consider it in the light of the concepts of non-extensive thermodynamics.

Generally, the modeling of the behavior of supersaturated fluids is conducted by assigning a specific form to the gaseous aggregate (spherical bubble, sessile bubble on a surface, in crevices of a material, etc.). The interfacial energy between the gas in the bubble and the fluid is then introduced, in equilibrium conditions, based on the Laplace's law. However, in natural systems, the gas/environment interfaces are often vague and do not permit to locate precisely the geometric boundaries of gaseous aggregate. Its form is most often imposed by the system 
itself. As example, let's take the case of a "gas bubble" formed in physiological tissue in the decompression of the diver. Where is located the border between the gaseous aggregate and its environment? What is the value of the interfacial area and the associated energy? How these parameters can be introduced in the relations of classical thermodynamics to describe the behavior of the system and its possible evolution?

In a set of previous works, we tackled this type of situation for condensed phases (solid and liquid) and showed that we could obtain conditions of equilibrium and evolution of the systems from thermodynamics based on non-extensive conventions. In these approaches formalized in 2004 [16], the phases' borders are not specified. The interfaces are supposed to be "fuzzy". We so treated the solubility of the small aggregates [17], their redox potential [18], their melting point [19], their surfactant properties in a solvent [20] or their wettabilities [21]. In these studies, we showed that, for non-extensive phases, the chemical potential, which is an intensive variable in the case of the unlimited phases, loses this property. Its value depends on the quantity of aggregates according to a power law.

In this paper, we will show that the properties of the gaseous aggregates in the presence of solutions supersaturated in gas can also be described by this type of approach. We shall thus propose a new formulation of the "equilibrium of super-saturation" of a gas in a liquid by taking into account the conventions of nonextensive thermodynamics. We shall find the classical descriptions and propose other possibilities of thinking. For example, we shall show that unlike the classical approaches, we can foresee conditions of degassing of fluids in conditions of sub-saturation. 


\section{Equilibrium conditions: the gas is confined; the liquid is an unlimited phase}

\subsection{Studied confined system and equilibrium conditions}

In the following development, we shall not try to determine the conditions of appearance of the gaseous aggregate, but the conditions for which this one is at thermodynamic equilibrium with its environment.

At temperature $\mathrm{T}$ and external pressure $\mathrm{P}$, the system schematized on the figure 1 is constituted by

- $\mathrm{n}_{\mathrm{i}}$ moles of pure gas $\mathrm{i}$ in a confined phase supposed to be non-extensive. The "shape" of the aggregate has not to be spherical [22]. It can be forced by its surrounding. To simplify the reasoning, we will suppose that the vapor pressure of the solvent in the confined gaseous phase is low enough to be neglected with regard to the internal pressure of the gas $\mathrm{P}_{\mathrm{NE}}$

- a phase (matrix) susceptible to solubilize the gas. This phase can be a pure liquid, a solution or a solvents mixture [23]. It will be considered as an unlimited phase. Its pressure is equal to the external pressure, $\mathrm{P}$.

- an interface between the gas and the matrix which is supposed to be fuzzy. 


\section{matrix}

\section{gas}

PNE

\section{$\mathbf{P}$}

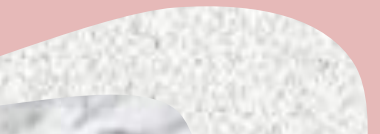

$P_{\text {NE }}$

\section{Fuzzy border}

Figure 1 : scheme of the studied system. The gas is the non-extensive phase in contact with the matrix through a fuzzy interface.

The gas $i$ contained in the confined phase is in equilibrium with the solute $i$ dissolved in the matrix with which it is in contact. The equilibrium condition of the system is translated by the equality of the chemical potential of $i$ under its forms gas, $\mu_{i(\text { gas })}^{P_{N E}}$ and solute, $\mu_{i(s o l)}^{P}$,

$$
\mu_{i(\text { gas })}^{P_{N E}}=\mu_{i(\text { sol })}^{P}
$$

The expressions of the chemical potentials in the two media are expanded

$$
\mu_{i(g a s)}^{*}+R T \ln \left\{f_{i}\right\}=\mu_{i(s o l)}^{\infty, P}+R T \ln \left\{a_{i}\right\}
$$

$\mu_{i(\text { gas })}^{*}$ is the standard chemical potential of the gas. This quantity does not vary with the temperature.

$\mu_{i(s o l)}^{\infty, P}$ is the standard chemical potential of the solute, referred to the behavior of infinite diluted solution. This quantity varies with the temperature and the pressure according to the following relation 


$$
\left(\frac{\partial \mu_{i(\text { sol })}^{\infty, P}}{\partial P}\right)_{T, n_{i}}=V_{i}^{\infty}
$$

$V_{i}^{\infty}$ is the partial molar volume of the gas in solution.

We define $\left\{f_{i}\right\}$ as the value of the gas fugacity. The latter is linked to the pressure of $i$ pure in the nonextensive phase by

$$
\left\{f_{i}\right\}=\lambda_{i}^{P_{N E}}\left\{P_{N E}\right\}
$$

$\lambda_{i}^{P_{N E}}$ is the fugacity coefficient of the gas at the pressure of the nonextensive phase.

$\left\{a_{i}\right\}$ is the value of the activity of $i$, in the concentration scale, referred to the behavior of infinite diluted solutions. Let's take the case of gases slightly soluble in liquids, for which values of activity and concentration of $i$ are equal

$$
\left\{a_{i}\right\}=\left\{C_{i}\right\}
$$

The equilibrium condition can be written as,

$$
\mu_{i(\text { gas })}^{*}+R T \ln \left\{f_{i}\right\}=\mu_{i(\text { sol })}^{\infty, P}+R T \ln \left\{C_{i}\right\}
$$

Since, the standard chemical potential of the solute $i$ varies with the pressure of the unlimited liquid phase, we can refer to an arbitrary pressure $\mathrm{P}^{\circ}$, for example, the pressure of 1 bar. If the value of the partial volume of $i$ is supposed to be independent of the pressure, the expression of the chemical potential of the gas become

$$
\mu_{i(s o l)}^{\infty, P}=\mu_{i(s o l)}^{\infty, P^{o}}+V_{i}^{\infty}\left(P-P^{o}\right)
$$

And then,

$$
\ln \left\{f_{i}\right\}=\frac{\mu_{i(\text { sol })}^{\infty, P^{o}}-\mu_{i(g a s)}^{*}}{R T}+\frac{V_{i}^{\infty}\left(P-P^{o}\right)}{R T}+\ln \left\{C_{i}\right\}
$$

With,

$$
\frac{\mu_{i(s o l)}^{\infty, P^{o}}-\mu_{i(g a s)}^{*}}{R T}=\ln K h_{i}^{o}
$$


$K h_{i}^{o}$ is the solubility constant of the system, it is a dimensionless extent. The concentration of the gas $i$ in the solution is linked to the nonextensive pressure of the gas in the confined phase by

$$
P_{N E}=\frac{P_{r e f} K h_{i}^{o}}{C_{r e f} \lambda_{i}^{P_{N E}}} C_{i} e^{\frac{V_{i}^{\infty}\left(P-P^{o}\right)}{R T}}=\frac{K_{i}^{o}}{\lambda_{i}^{P_{N E}}} C_{i} e^{\frac{V_{i}^{\infty}\left(P-P^{o}\right)}{R T}}
$$

$P_{r e f}$ and $C_{r e f}$ are the pressure and concentration taken as units to express the adopted reference systems. $K_{i}^{o}$ is a dimensioned magnitude. This relation is identical to the one proposed by Krichevsky and Kasarnovsky [24] in 1935.

The problem is now to link this magnitude to the external pressure, P. For that, we used the results of the nonextensive thermodynamics [16].

\subsection{Solubility of a gas forming a nonextensive phase}

The main advance we had realized in 2004 , was to show that the pressure in a nonextensive phase is different from the ambient pressure since an energy is associated with the interface, whatever "the shape" of the phase is, even if this one is indistinct. In the general case, we demonstrate that, for a system involving only a single interface with its environment, the difference of pressure between the nonextensive phase $\mathrm{P}_{\mathrm{NE}}$ and the controllable external pressure, $\mathrm{P}$, can be written as

$$
P_{N E}-P=\tau \frac{d \chi}{d V}
$$

$V$ is the volume of the confined phase. For condensed systems, it is an extensive extent, i.e., whose value varies proportionally with the system mass.

$\chi$ is an «extensity ». It is a nonextensive magnitude characteristic of the fuzzy interface. In the general case, the value of $\chi$ does not vary proportionally with the mass of the considered system (contrary to the extensive magnitude). The variable $\tau$ is the intensive magnitude 
associated to the extensity $\chi$. Its value can be positive or negative. It depends on the gas nature and the matrix. For condensed phases (solids and liquids), we set down by convention that $\chi$ is an Euler's function of order $\mathrm{m}(1 \geq m \geq 0)$ of the number of moles, $n_{i}$. The value of $\chi$ increases with the system mass. Since the volume is an extensive extent (Euler's function of order 1 of the mass), $\chi$ is a homogeneous function of order $m$ of the volume. Eq. 11 becomes,

$$
P_{N E}-P=\tau \frac{d \chi}{d V}=m \tau \frac{\chi}{V}
$$

As is shown in our previous works, this relation permits to simply recover the expression of Laplace's law for a spherical system of radius $r$. the extensity $\chi$ is identified with the sphere area which is an Euler's function of order $2 / 3$ of the volume and $\tau$ with the superficial tension.

$$
P_{N E}-P=\gamma \frac{d A}{d V}=\gamma \frac{d\left(4 \pi r^{2}\right)}{d\left(\frac{4}{3} \pi r^{3}\right)}=\frac{2 \gamma}{r}=\frac{2}{3} \gamma \frac{A}{V}
$$

The approach we followed for condensed phases must be adapted to gases because these are compressible. As a result the volume of a gaseous aggregate is not any more an extensive magnitude; this can be easily checked for a sphere containing $n_{i}$ moles of gas the internal pressure of which is fixed by Laplace's law.

$$
P_{N E} V-P V=n_{i} R T-P V=\frac{2 \gamma}{r} V
$$

By taking into account the geometrical characteristics of the spherical bubble,

$$
\frac{n_{i} R T}{P}=V+\frac{2 \gamma}{P}\left(\frac{4 \pi}{3}\right)^{1 / 3} V^{2 / 3}
$$

We see that if the number of moles $n_{i}$ is multiplied by $\lambda$, at constant external pressure $P$, the volume is not multiplied by $\lambda$. 
Thus, for gases, we shall be restricted the original convention of the nonextensive thermodynamics which is that the extensity $\chi$ is a homogeneous function of order $m$ of the aggregate volume (eq. 12). We shall not try to link the magnitudes ( $\chi$ and $V$ ) to the contents of the system from geometrical relations characteristic of particular shapes.

In our previous studies, we showed (especially in reference [21]) that when the nonextensive phase, of volume $V$, involves several interfaces with its surrounding, the pressure difference is the sum of the contributions of each interface, $\mathrm{j}$,

$$
P_{N E}-P=\tau \frac{d \chi}{d V}=\sum_{j} m_{j} \tau_{j} \frac{\chi_{j}}{V}
$$

The expression of $\mathrm{P}_{\mathrm{NE}}$ can be introduced in eq. 10

$$
P=\frac{K_{i}^{o}}{\lambda_{i}^{P_{N E}}} C_{i} e^{\frac{V_{i}^{\infty}\left(P-P^{o}\right)}{R T}}-\sum_{j} m_{j} \tau_{j} \frac{\chi_{j}}{V}
$$

This relation links the solubility of a gas contained in a confined phase at the fixed external pressure. It is "Henry's law" generalized to small gaseous systems in the presence of a matrix susceptible to solubilize the gas.

To continue the discussion more easily, we adopt simplifying conditions.

\subsection{Simplifying conditions}

Let take the simple case where the variations of pressure are weak enough such as $e^{\frac{V_{i}^{\infty}\left(P-P^{o}\right)}{R T}}=1$. This approximation is justified by the very low value of the partial molar volume of the gases [25-28] in the liquids. Besides, the gas is supposed to behave as perfect gas $\left(\lambda_{i}^{P_{N E}}=1\right)$. In this framework, eq. 17 can be simplified,

$$
P=K_{i}^{o} C_{i}-\sum_{j} m_{j} \tau_{j} \frac{\chi_{j}}{V}
$$


This relation, which considers several interfaces, links the concentration of the gas solubilized in the matrix and the external pressure imposed on the system. The solubility of the gas in this condition can be compared its solubility, $C_{i}^{o}$ if the phases would be unlimited, $P=K_{i}^{o} C_{i}^{o}$

$$
\mathcal{R}=\frac{C_{i}}{C_{i}^{o}}=1+\frac{1}{P V} \sum_{j} m_{j} \tau_{j} \chi_{j}
$$

$\mathcal{R}$ is the rate of supersaturation. We can clarify this relation by introducing the fact that the variables of extensities, $\chi$, are Euler's functions of order $\mathrm{m}$ of the volume of the confined phase, by convention. We ascribe to them a shape of "power law", such as

$$
\chi_{j}=\alpha_{j} V^{m_{j}}
$$

$\alpha_{\mathrm{j}}$ is a constant characteristic of the considered interface and characterized by $\chi_{j}$. This leads to

$$
P=K_{i}^{o} C_{i}-\sum_{j} m_{j} \tau_{j} \alpha_{j} V^{m_{j}-1}
$$

This relation shows that there is no proportionality between $P$ and $C_{i}$ in the case where the gas is constituted by the confined phase. The solubility of the gas depends on the volume of the gaseous aggregate according to a power law. The relation between the solubilities is written as

$$
\mathcal{R}=\frac{C_{i}}{C_{i}^{o}}=1+\frac{1}{P} \sum_{j} m_{j} \tau_{j} \alpha_{j} V^{m_{j}-1}
$$

Besides, we notice that according to the sign of the variables of intensity $\tau_{j}$, which can be positive or negative, the concentration, $C_{i}$, can be higher or lower to $C_{i}^{o}$, what allows envisaging the existence of systems for which there is presence of gaseous aggregates in equilibrium with the gas solubilized for concentrations lower than the saturation.

The limits of eq. 22 allow recovering the properties of unlimited systems. When the volume of the aggregates becomes big, the phase gas loses its nonextensive properties. For $m<1$, 
$m_{j} \tau_{j} \alpha_{j} V^{m_{j}-1} \rightarrow 0$ what implies that $C_{i}$ is equal to $C_{i}^{o}$. We shall discuss afterward the particular case in which $\mathrm{m}_{\mathrm{j}}=1$.

\subsection{Solubilities of gases in confined phase}

Now, let imagine various cases of applications of these relations and consider the particular case of the systems presenting a single interface with the matrix. The application of eq. 22 to a single interface allows examining the behavior of a gaseous aggregate in solution in equilibrium with the dissolved gas.

$$
\mathcal{R}=\frac{C_{i}}{C_{i}^{o}}=1+\frac{1}{P} m \tau \alpha V^{m-1}
$$

In the case of a spherical aggregate, of radius $r$, this relation allows to get the relation used by numerous authors to express the solubility of bubbles

$$
\mathcal{R}=\frac{C_{i}}{C_{i}^{o}}=1+\frac{1}{P} \frac{2 \gamma}{r}
$$

However, its experimental check was rarely undertaken. Recently, Ohgaki et al. [29] published a study on the behavior of nano-bubbles of various gases of radius of $50 \mathrm{~nm}$ in water at $298 \mathrm{~K}$. For nitrogen, these authors show that they obtain suspensions of stable bubbles during more than two weeks, containing until $600 \mathrm{~cm}^{3}$ of confined gas (standard condition) by liter of solution. Even if it is thermodynamically "unstable" in the sense where the free energy is maximum, the equilibrium of the system constituted by bubbles in the medium is not quickly destroyed by the fluctuations of the environment. The nitrogen solubility in the solution is 36 times higher than the solubility in unlimited phases. The experimental value of the internal pressure was found equal to 63 bars, while the use of the eq. 24 with a superficial tension equal to $7210^{-3} \mathrm{~N} \mathrm{~m}^{-1}$ leads to a value of 30 bars, is approximately half of that determined. The value of the internal pressure thus exceeds the 
calculated one, while it is shown that the superficial tension of the bubble decreases when its curvature increases [30]. The discrepancy between calculated and experimental values can have various origins of which an estimation too much idealized of the shape and the size of bubbles. The micrographies show that the aggregates are not perfect spheres and the opinion of the authors is that the suspension of nano-bubbles is not strictly homogeneous. But if we preserve the hypothesis that Laplace's law applies to these systems, then it is necessary to imagine that all the nano-bubbles, even imperfect, behave as a set of spheres of radius of 50 $\mathrm{nm}$.

It seems more reasonable to envisage that the law of Laplace does not apply to these objects, using the argument that their geometrical border is badly defined.

This remark had already been made by Bowers [31] in 1996. The latter supposed that the interfaces for nano-bubbles were vague, and that there was a domain of transition between the gas and the liquid (blobs). Eq. 12, applied to a single interface, allows expressing the pressure difference between the inside of this type of aggregate and the environment according to its volume by a power law.

$$
P_{N E}-P=m \tau \alpha V^{m-1}
$$

The gas solubility is then given by

$$
\mathcal{R}=\frac{C_{i}}{C_{i}^{o}}=1+m \tau \frac{\chi}{P V}=1+\frac{m \tau \alpha V^{m-1}}{P}
$$

The validity of these relations has to be checked from further studies as those proposed by Ohgaki et al.[29], by determining the gas solubility for various nanobubbles sizes and external pressure, that obviously raises the problem of controlled obtaining of such systems. Anyway, eq. 26 shows that for a given rate of supersaturation, there is a situation of (unstable) equilibrium for which the mass of the gaseous aggregate is perfectly defined, in the conditions imposed by its environment, in particular for its shape which cannot be spherical. It is 
surprising that, in some analyses, the authors wonder on the stability of bubbles using the argument that their internal pressures are much higher than the surrounding pressure. This pressure difference is the reason for which the system is at equilibrium. So this pressure difference cannot be regarded as the origin of a thermodynamic force therefore disequilibrium. The affinity of such a system is then null.

$$
\mathcal{A}=\sum v_{i} \mu_{i}=\mu_{i}^{P_{N E}}-\mu_{i}^{P}=0
$$

The only thermodynamic force at stake involves the chemical potentials.

However, the equilibrium corresponding to a maximum of free energy, these systems should evolve either toward the solubilization of aggregates or toward their increase due to the fluctuations in environmental parameters such as temperature. This does not seem to be the case, suggesting a small change in the Gibbs energy with the radius around the critical radius. The existence of nanobubbles solutions [32-35] and stable encapsulated microbubbles [36, 37] is one example. Different authors suggest several assumptions to justify this property [13, 38]. The molecular dynamics simulation can provide on this subject some precious elements of thinking [39].

The originality of eq. 26 is to allow considering the creation of bubbles in a sub-saturation medium when $\tau$ is negative.

We had encountered this kind of situation when analyzing the values of the melting points of nano-systems [19]. The melting point of a particle of the same metal can decrease $(\tau>0)$ when its size decreases, or on the contrary, increase $(\tau<0)$ depending on the environment of non-extensive phase. In the case of particles embedded in a matrix, it increases.

In this case, the possibility of observing the creation of gas clusters in equilibrium with the solution cannot be dismissed even at concentrations below saturation.

As $\tau$ can be negative, we can then consider that the pressure in the aggregates is lower than this of the environment, but with the condition $P>-m \tau \alpha V^{m-1}$, since the term $P_{N E}$ 
(occurring in a logarithm in the expression of the gas chemical potential) must be positive. The problem is different if one considers a non-extensive condensed phase. Internal pressures of the aggregates can then be negative. This will be detailed in the discussion.

Eq.26 allows considering different system behaviors depending on the chosen value of $m$. We showed that with $m=2 / 3$, we formally found the behavior of spherical bubbles. Other values are interesting, such as $m=1$. In this case, when the number of moles of the aggregate is multiplied by $\lambda$, the extensity variable $\chi$ and the volume of non-extensive phase are also multiplied by $\lambda$. Then, the solubilization law takes the form

$$
\{P\}=K_{i}^{o}\left\{C_{i}\right\}-\tau \alpha
$$

This case corresponds for example to a non-extensive phase which is replicated when the amount of gas increases. The discrepancy between the concentration of the supersaturated solution and the concentration at saturation is constant and does not vary with the amount of gas.

An illustration of this behavior is given in the paper of Bowers et al. [40] which shows a "super-Henry's Law" for the solubility of oxygen produced by the catalyzed decomposition of iodine by hydrogen peroxide. In this study, the plot of the concentration of dissolved gas according to the pressure is parallel to the Henry's Law, since it corresponds to the following relation

$$
\left\{C_{i}\right\}=\frac{\{P\}}{K_{i}^{o}}+\frac{\tau \alpha}{K_{i}^{o}}
$$

In the work of Bowers et al. [31, 40], this behavior is considered as particular case of the classical nucleation theory $(\mathrm{CNT})$, for which the rate of nucleation is constant. 


\section{Discussion}

The previous relations form a framework of thoughts which permit to examine some works and to imagine others on the behaviors of bubbles in solution and more generally, on the conditions of solubilization equilibrium of gases in liquids in confined spaces.

\section{Use of Kelvin's relation}

In some studies, the solubility in liquids of gases contained in bubbles is discussed from Kelvin's relation, by simply changing the sign before the term relative to the contribution of the interfacial energy. The equilibrium between a liquid and its vapor is treated by the same way. Thus, Defay [41] suggested the following relation to express the variations in vapor pressure of a liquid in equilibrium with bubbles, with the conclusion that the smaller is the vapor pressure of the liquid, the stronger is the curvature.

$$
\ln \frac{P_{i}}{P_{i}^{o}}=-\frac{2 \gamma}{r} \frac{V^{*}}{R T}
$$

$P_{i} \quad$ and $P_{i}^{o}$ are respectively the vapor pressures of the liquid in the bubble and in the unlimited gas phase, $V^{*}$ is the bubble volume.

This relation was recently used by Castellanos et al.[42] to show the need to consider the decrease of the surface tension of bubbles with their radius for much higher size ranges (of about $4 \log$ units of the radius) than those assumed for the drops.

In our opinion, eq. 30 is not suitable to describe the behavior of a gas bubble in an unlimited environment. In fact, it is simply shown that vapor pressure in a bubble is invariant with the radius of the bubble, which has been further confirmed by simulation[43].

Consider a liquid - gas equilibrium, for which the pressure of the liquid phase is $P$ and that of the gas, $P_{b}$. The equilibrium condition is written

$$
\mu_{i(\text { gas })}^{P_{b}}=\mu_{i(l i q)}^{P}
$$

Both terms are expanded 


$$
\mu_{i(g a s)}^{*}+R T \ln \left\{P_{b}\right\}=\mu_{i(l i q)}^{*}
$$

Which involves that

$$
\ln \left\{P_{b}\right\}=\frac{\mu_{i(l i q)}^{*}-\mu_{i(g a s)}^{*}}{R T}=\text { constant }
$$

The value of vapor pressure in a bubble, put in an unlimited solution at pressure $P$, is then constant. It does not depend on the curvature radius of the bubble.

We share the skepticism of Seddon et al.[44] about the work of Colaço et al.[45] on the inappropriate use of the Kelvin relation. The latter applies only to limited condensed phases. This can be merely shown by considering the solubility of a gas (in an unlimited phase) in a liquid aggregate. This is the opposite case to the one discussed above.

Using the same formalism as before, the equilibrium condition concerns the equality of chemical potentials of $i$ in its gas form, $\mu_{i(g a s)}^{P}$ and as solute, $\mu_{i(l i q)}^{P_{N E}}$,

$$
\mu_{i(\text { gas })}^{P}=\mu_{i(\text { liq })}^{P_{N E}}
$$

The expressions are expanded in the framework of the nonextensive thermodynamics

$$
\mu_{i(g a s)}^{*}+R T \ln \{P\}=\mu_{i(s o l)}^{P_{V E}}-\mu_{i(s o l)}^{P}+\mu_{i(s o l)}^{P}=m \tau \frac{\chi}{V}+\mu_{i(s o l)}^{\infty}+R T \ln \left\{C_{i}\right\}
$$

The relation linking the gas solubility in the confined phase to the pressure of the unlimited phase is

$$
\ln \{P\}=m \tau \frac{\chi}{R T V}+\frac{\mu_{i(s o l)}^{\infty}-\mu_{i(g a s)}^{*}}{R T}+\ln \left\{C_{i}\right\}
$$

which can be also written by introducing the solubility constant, $K h_{i}^{0}$,

$$
\ln \frac{\{P\}}{\left\{C_{i}\right\}}=m \tau \frac{\chi}{R T V}+\ln K h_{i}^{o}
$$

The comparison of the gas solubility in the liquid in unlimited phase, $C_{i}^{o}$, and in condensed phase is written as 


$$
\ln \frac{\left\{C_{i}\right\}}{\left\{C_{i}^{o}\right\}}=-m \tau \frac{\chi}{R T V}=-m \tau \frac{\alpha V^{m-1}}{R T}
$$

When $\tau$ is positive (this is the case of water drops) the gases are less soluble in the condensed liquid phase as the volume of the aggregates decreases. For a spherical aggregate $(m=2 / 3, \tau=$ $\gamma)$, one obtains

$$
\ln \frac{\left\{C_{i}\right\}}{\left\{C_{i}^{o}\right\}}=-\frac{2}{3} \gamma \frac{4 \pi r^{2}}{R T \frac{4}{3} \pi r^{3}}=-\frac{1}{R T} \frac{2 \gamma}{r}
$$

Considering the fogs as systems where water is in the form of spherical liquid drops, the application of eq.39 provides an under-saturation of the aqueous phase. This property is not verified for many systems where there are sometimes supersaturations. Such behavior cannot be justified by classical thermodynamics. However, in the context of non-extensive thermodynamics we can assume that the natural environment that can be fog or cloud mades of liquid aggregates whose boundaries are ill-defined and for which the intensity variable $\tau$ is negative. In this case, it is possible to observe gas supersaturation in the aqueous entities with an amplification of the phenomenon when the aggregate size decreases [46, 47].

\section{Conclusion}

In this paper, we have shown that the use of non-extensive thermodynamics' concepts allowed formalizing in a very general way, even in complex systems, the equilibrium solubility of gases contained in bubbles, or in gas aggregates, in the presence of supersaturated solutions. Besides introducing new relationships and discussion of the applicability of other (Kelvin), the main contributions of this work were to show

- that the application of Laplace's law to the interface gas / solution at nanometric state is unlikely. This interface is probably geometrically ill-defined and can be described as a fuzzy interface. 
- the possibility of considering the presence of gaseous aggregates in equilibrium with the solution even when the latter is under-saturated.

Although the relevance of the overall relations could not be verified by lack of experimental data, it remains true that the proposed relations are a way of thinking to interpret the behavior of these complex systems, to imagine or to justify other. This is the case of the concept of negative pressure which was presented in different works [48-50] and variously commented $[51,52]$. In non-extensive thermodynamics, nothing opposes to a negative value of the nonextensive pressure for condensed phases, since we consider negative intensities, $\tau$,

$$
P_{N E}=P+\sum_{j} m_{j} \tau_{j} \frac{\chi_{j}}{V}<0
$$

The increases in melting temperatures, observed for coated metallic clusters when their size decreases [19], fall within this property.

The concepts of non-extensive thermodynamics apply to a wide scope in confined condensed phases. In the nanometric scale, classical thermodynamics is undoubtedly no more applicable $[53,54]$.

The nano-thermodynamics is probably not extensive. 


\section{References}

[1] M. Hamada, D. Laporte, N. Cluzel, K. T. Koga, T. Kawamoto, Bull. Volcanol. 72 (6) (2010) 735-746.

[2] S. Yoshimura, M. Nakamura, Chem. Geol. 276 (1-2) (2010) 18-28.

[3] G. Liger-Belair, G. Polidori, P. Jeandet, Chem. Soc. Rev. 37 (11) (2008) 2490-2511.

[4] A. Agarwal, W. J. Ng, Y. Liu, Chemosphere 84 (9) (2011) 1175-1180.

[5] S. J. Yang, A. Duisterwinkel, Langmuir 27 (18) (2011) 11430-11435.

[6] N. Matsuki, S. Ichiba, T. Ishikawa, O. Nagano, M. Takeda, Y. Ujike, T. Yamaguchi, Eur. Biophys. J. 41 (6) (2012) 571-578.

[7] B. R. Wienke, Comput. Biol. Med. 40 (3) (2010) 252-260.

[8] M. A. Chappell, S. J. Payne, Respir. Physiol. Neurobiol. 153 (2) (2006) 166-180.

[9] M. A. Chappell, S. J. Payne, Respir. Physiol. Neurobiol. 152 (1) (2006) 100-114.

[10] R. Ball, S. L. Schwartz, Clin. Pharmacokinet. 41 (6) (2002) 389-402.

[11] D. E. Yount, D. C. Hoffman, Aviat. Space Environ. Med. 57 (2) (1986) 149-156.

[12] D. E. Yount, R. H. Strauss, J. Appl. Phys. 47 (11) (1976) 5081-5089.

[13] N. F. Bunkin, S. O. Yurchenko, N. V. Suyazov, A. V. Shkirin, J. Biol. Phys. 38 (1) (2012) 121-152.

[14] J. Mitrovic, Chem. Eng. Sci. 55 (12) (2000) 2265-2270.

[15] S. D. Lubetkin, Langmuir 19 (7) (2003) 2575-2587.

[16] M. Turmine, A. Mayaffre, P. Letellier, J. Phys. Chem. B 108 (49) (2004) 18980-18987.

[17] P. Letellier, A. Mayaffre, M. Turmine, J. Phys.: Condens. Matter 19 (43) (2007) 4362299.

[18] P. Letellier, A. Mayaffre, M. Turmine, J. Phys. Chem. C 112 (32) (2008) 12116-12121.

[19] P. Letellier, A. Mayaffre, M. Turmine, Phys. Rev. B 76 (4) (2007) 045428.

[20] P. Letellier, A. Mayaffre, M. Turmine, J. Colloid Interface Sci. 321 (1) (2008) 195-204.

[21] P. Letellier, A. Mayaffre, M. Turmine, J. Colloid Interface Sci. 314 (2) (2007) 604-614.

[22] S. L. Meadley, F. A. Escobedo, J. Chem. Phys. 137 (7) (2012) 074109-074109.

[23] A. Habich, W. Ducker, D. E. Dunstan, X. H. Zhang, J. Phys. Chem. B 114 (20) (2010) 6962-6967.

[24] I. R. Krichevsky, J. S. Kasarnovsky, J. Am. Chem. Soc. 57 (1935) 2168-2171.

[25] Osulliva.Td, N. O. Smith, J. Phys. Chem. 74 (7) (1970) 1460-\&.

[26] T. L. Zhou, R. Battino, J. Chem. Eng. Data 46 (2) (2001) 331-332.

[27] I. Cibulka, A. Heintz, Fluid Phase Equilib. 107 (2) (1995) 235-255.

[28] R. Sun, W. X. Hu, Z. H. Duan, J. Sol. Chem. 30 (6) (2001) 561-573.

[29] K. Ohgaki, N. Q. Khanh, Y. Joden, A. Tsuji, T. Nakagawa, Chem. Eng. Sci. 65 (3) (2010) 1296-1300.

[30] R. C. Tolman, J. Chem. Phys. 17 (3) (1949) 333-337.

[31] P. G. Bowers, K. BarEli, R. M. Noyes, J. Chem. Soc.-Faraday Trans. 92 (16) (1996) 2843-2849.

[32] P. Attard, M. P. Moody, J. W. G. Tyrrell, Phys. A 314 (1-4) (2002) 696-705.

[33] K. Kikuchi, A. Ioka, T. Oku, Y. Tanaka, Y. Saihara, Z. Ogumi, J. Colloid Interface Sci. 329 (2) (2009) 306-309.

[34] K. Kikuchi, S. Nagata, Y. Tanaka, Y. Salhara, Z. Ogumi, J. Electroanal. Chem. 600 (2) (2007) 303310.

[35] F. Y. Ushikubo, T. Furukawa, R. Nakagawa, M. Enari, Y. Makino, Y. Kawagoe, T. Shiina, S. Oshita, Colloid Surf. A 361 (1-3) (2010) 31-37.

[36] B. Krasovitski, E. Kimmel, Ultrason. 44 (2) (2006) 216-220.

[37] A. Katiyar, K. Sarkar, P. Jain, J. Colloid Interface Sci. 336 (2) (2009) 519-525.

[38] J. R. T. Seddon, D. Lohse, W. A. Ducker, V. S. J. Craig, Chemphyschem 13 (8) (2012) 2179-2187.

[39] J. H. Weijs, J. R. T. Seddon, D. Lohse, Chemphyschem 13 (8) (2012) 2197-2204.

[40] P. G. Bowers, C. Hofstetter, C. R. Letter, R. T. Toomey, J. Phys. Chem. 99 (23) (1995) 9632-9637.

[41] R. Defay, Surface tension and adsorption. Longmans, Green and Co: London, 1966.

[42] A. J. Castellanos, J. Toro-Mendoza, M. Garcia-Sucre, J. Phys. Chem. B 113 (17) (2009) 5891-5896.

[43] M. Matsumoto, K. Tanaka, Fluid Dyn. Res. 40 (7-8) (2008) 546-553. 
[44] J. R. T. Seddon, H. J. W. Zandvliet, Surface Science 604 (3-4) (2010) 476-477.

[45] R. Colaco, A. P. Serro, B. Saramago, Surf. Sci. 603 (18) (2009) 2870-2873.

[46] W. Winiwarter, H. Fierlinger, H. Puxbaum, M. C. Facchini, B. G. Arends, S. Fuzzi, D. Schell, U. Kaminski, S. Pahl, T. Schneider, A. Berner, I. Solly, C. Kruisz, J. Atmos. Chem. 19 (1-2) (1994) 173-188.

[47] B. Ervens, P. Herckes, G. Feingold, T. Lee, J. L. Collett, S. M. Kreidenweis, J. Atmos. Chem. 46 (3) (2003) 239-269.

[48] L. Mercury, Y. Tardy, C.R. Acad. Sci. Paris 324 (11) (1997) 863-873.

[49] L. Mercury, M. Azaroual, H. Zeyen, Y. Tardy, Geochim. Cosmochim. Acta 67 (10) (2003) 17691785.

[50] M. M. Conde, C. Vega, G. A. Tribello, B. Slater, J. Chem. Phys. 131 (3) (2009) 034510.

[51] J. V. Walther, Geochim. Cosmochim. Acta 68 (12) (2004) 2771-2773.

[52] L. Mercury, Y. Tardy, Geochim. Cosmochim. Acta 68 (12) (2004) 2775-2780.

[53] P. Mohazzabi, G. A. Mansoori, J. Comput. Theor. Nanosci. 2 (1) (2005) 138-147.

[54] G. R. Vakili-Nezhaad, G. A. Mansoori, J. Comput. Theor. Nanosci. 1 (2) (2004) 227-229. 